\title{
Evidence on collaboration of traditional and biomedical practitioners in the management of antimicrobial resistance in sub-Saharan Africa over 15 years: A systematic review protocol
}

\section{AGANZE GLOIRE-AIME MUSHEBENGE ( $\nabla$ aganzedar@Gmail.com )}

University of KwaZulu-Natal College of Health Sciences https://orcid.org/0000-0001-8745-8097

\section{MUKANDA Gedeon KADIMA}

University of KwaZulu-Natal College of Health Sciences

TIVANI Phosa MASHAMBA-THOMPSON

University of Pretoria Faculty of Health Sciences

MANIMBULU NLOOTO

University of Limpopo Faculty of Health Sciences

\section{Protocol}

Keywords: Collaboration, Traditional Health Practitioners, Drug-resistance, traditional medicine, Absence, Conventional antimicrobial, Prevention, Effective, sub-Saharan Africa

Posted Date: January 19th, 2021

DOI: https://doi.org/10.21203/rs.2.22650/v2

License: (c) (i) This work is licensed under a Creative Commons Attribution 4.0 International License.

Read Full License 


\section{Abstract}

\section{Background}

The overuse of prescribed antimicrobials, concurrent use of traditional medicine, and prescribed antimicrobials have led to antimicrobial resistance. The absence of collaboration between traditional health practitioners and biomedically trained healthcare professionals can contribute to antimicrobial resistance, treatment failure, overdose, toxicity, and misadministration. This scoping review explores the evidence on collaboration between traditional health practitioners and biomedically trained healthcare professionals to reduce antimicrobial resistance and treatment failure in bacterial and viral diseases.

\section{Methods/design}

We will search for electronic databases such as Science Direct, Google Scholar, PubMed, MEDLINE via EBSCOhost. We will also search reference lists of included studies. A two-stage mapping procedure will be carried out. Stage one (1) will consist of the title, abstracts, and full article screening, respectively. A pilot screening form guided by the defined eligibility criteria will be used. Stage two (2), data will be extracted from the included studies. Two reviewers will conduct parallel screening and data extraction. Mixed methods appraisal tool (MMAT) will be used to assess the quality of the included studies. NVIVO version 11 will be employed to aid pertinent thematic analysis. The outcomes of interest will be as follows: Primary outcome will be preventing and reducing antimicrobial resistance. The secondary effect is the effective collaboration between traditional healthcare practitioners and biomedically healthcare professionals.

\section{Discussion}

This review anticipates uncovering pertinent publications reporting the evidence of collaboration between traditional health practitioners and biomedically trained healthcare professionals to reduce antimicrobial resistance in sub-Saharan Africa. The sum-up of evidence acquired from the included studies will help guide future research. The result of the study will be print and electronically exposed.

\section{Systematic review registration ID: PROSPERO CRD42017072952}

\section{Background}

Traditional treatment or indigenous health system cannot be considered lower quality than the mainstream healthcare system. In contrast, it is thought to be desirable and needful in treating a range of several health troubles or difficulties that the mainstream healthcare system fails to cure sufficiently[1, 2]. In the view of Qi and Kelley [2], traditional herbal mixtures are a reliable, believable, and dignified source of health care. Bacterial and viral infections are currently common; some pathogens have become resistant to multiple antimicrobials classes [3]. Microbial adaptation allows microbes to persist despite 
the presence of an antibiotic or antiviral agent; this reduces the potential human health benefit derived from antibiotics and antiviral medicines[4, 5].

The ratio of traditional health practitioners and biomedically trained professionals to the general population in sub-Saharan Africa is approximately 1:500 and 1:40000, respectively [6]. In addition to their modern biomedical treatment, up to 70 percent of South Africans are consulted by an estimated 200000 indigenous traditional healers [6, 7]. Approximately 27 million South Africans, including people living with HIV/AIDS and bacteria-infected people, depend on traditional medicine(TM) for their primary health care needs [8]. STIs such as Tshofela/drop (gonorrhea), Thosola (syphilis), some other specific bacterial infections, and assumed HIV/AIDS is the most commonly treated conditions and problems by THPs [9]. This is probably due to the excellent accessibility of plants, affordability, the confidentiality of health information between the patient and practitioner, and the high cost of synthetic

medicines [10]. Furthermore, consumers believe that certain infections such as acne, warts, shingles, and STIs cannot be treated with western medicine by BHPs but rather by THPs [11, 12]. Medicinal plants are effective, cheap, readily available, and used for cultural reasons.

In South Africa, antimicrobial resistance (AMR) is hugely prevalent. Some bacteria and viruses are becoming so resistant that there is either only antimicrobial of last resort or infections are untreatable [13]. AMR in South Africa is driven by many factors such as the careless use of prescribed antimicrobials, the use of traditional medicine (TM) currently not regulated, the lack of collaboration between traditional health practitioners(THPs) and biomedically trained healthcare professionals(BHPs), with the result of treatment failure, misadministration, interactions and toxicity [14]. To conceptualize the conditions for collaboration between the two systems, Pretorius [15] proposed the Biomedical/Traditional Medical Relationship's analogical model. This model shows clearly that each aspect of the traditional medicine system may be related to the western medicine system, such as mutual referral. Besides, the WHO have adopted the strategies to ensure the integration of collaboration between research institutions and THPs based on research and management of patients; and between THPs and BHPs in human immunodeficiency virus and acquired immune deficiency syndrome (HIV/ AIDS) prevention and sexually transmitted infections (STIs)/tuberculosis programs [16].

Although there is an increase in TM's use, it is not currently regulated, with the result of enhancement of the activities of standard medicines when used concurrently with TM [17]. Nascimento et al. reported that the danger of misadministration, drug interactions, overdose, toxicity, especially the problem of drug resistance and treatment failure, can occur when TM and prescribed antimicrobials are simultaneously and indiscriminately used [18]. This review aims to map the evidence on the collaboration between THPs and BHPs to reduce antimicrobial resistance in sub-Saharan Africa.

\section{Methods/design}

\section{Scoping review framework}


The authors will conduct a scoping review of peer-reviewed literature on the following specific points: concurrent use of traditional medicines and prescribed antimicrobials, the collaboration between THPs and BHPs, and treatment of bacterial and viral diseases. The scoping review method's selection was to make easy the mapping of the topic under study and build evidence around the related to the subject [19]. This review will use the framework developed by Arksey and O'Malley [20]. This framework stipulated the following steps (a) identification of research questions, (b) identification of relevant studies, (c) charting the data, and (d) collation, summary, and report of findings.

\section{Identifying the research questions}

The general research question of this study is, "What is the evidence of collaboration between THPs and BHPs in the reduction of antimicrobial resistance among people living with infectious diseases in subSahara African countries?"

The specific research questions to answer the general question are as follows:

1. What is the evidence of TM's concurrent use, prescribed ATB, and ARVs medicine for viral and bacterial infections?

2. What is the evidence of the perceptions of TPHs and BHPs about the interaction between TM and prescribed ATB and ARV medicine for viral and bacterial infections?

3. Is there a bidirectional referral of patients between THPs and BHPs to manage bacterial and viral diseases?

\section{Eligibility of research questions}

The study will use an amended Population, Intervention, Comparison, Outcomes, and Study setting (PICOS) framework to evaluate research questions' eligibility (Table 1).

Table 1. PICOS framework for determination of eligibility of review question

\begin{tabular}{|l|l|}
\hline Criteria & Determinants \\
\hline Population & THPs and BHPs \\
\hline Intervention & Collaboration between traditional and biomedically healthcare workers \\
\hline Comparison & Absence of collaboration between THPs and BHPs \\
\hline Outcomes & $\begin{array}{l}\text { Primary outcome: Prevention and reduction of antimicrobial resistance } \\
\text { Secondary outcome: Effective collaboration }\end{array}$ \\
\hline Setting & Sub-Sahara Africa. \\
\hline
\end{tabular}




\section{Identification of relevant studies}

Studies that utilize mixed methods, qualitative and quantitative, published in peer-reviewed journals and university research spaces, thesis and dissertations, conference papers, and government desks will be assessed as part of the grey literature. Collected data will be from January 2005 to January 2020, addressing the above research questions. Different types of study designs will be used during the process of screening data. Authors will conduct an electronic search from the following databases: Science Direct, Scopus, Web of Science and Embase, Google Scholar, PubMed, and MEDLINE via EBSCOhost. Authors will explore internet sites such as the World Health Organization (WHO) and government internet sites for reports and policies on the collaboration of healthcare workers, measures on antimicrobial resistance, safe use of traditional medicine, and concurrent use of traditional medicine prescribed medicines. Through "Cited by," other articles will also be searched in the reference lists of selected papers. The search keywords will include Collaboration, Traditional Health Practitioners, Drug-resistance, traditional medicine, Absence, Conventional antimicrobial, Prevention, Effective, and sub-Saharan Africa.

\section{Study selection}

To be sure that the included studies have the specific information according to the eligibility criteria, they should respond to the evidence of collaboration between THPs and BHPs in reducing antimicrobial resistance and treatment failure in bacterial and viral diseases.

\section{Inclusion criteria}

For the inclusion of publications in this study, they should match with the undermentioned criteria:

1. There will be no language restriction in the inclusion of studies. The authors will request English or French versions of Italian, German, Chinese, and Portuguese exciting materials.

2. Focus on strategies of collaboration between THPs and BHPs.

3. Publications from January 2005 to January 2020.

4. Report on cases of treatment failure, drug-interactions, drug-resistance, and antimicrobial stewardship.

5. Publications on the use of traditional medicine in conjunction with prescribed antimicrobials by community members of 18 years and above.

6. Reports on death cause worldwide, with particular emphasis on sub-Saharan Africa.

\section{Exclusion criteria}

Studies will be excluded if they meet the following characteristics: 
- Articles published before 2005 and after January 2020.

- Articles that they do not report on TM's use for the management of bacterial and viral diseases.

- Articles that report on other diseases than infectious diseases.

\section{Search strategy}

A pilot study will be carried out to check the chosen studies' appropriateness, keywords, and databases. Selected articles will be shared between two reviewers using research manager software such as Endnote library. According to the eligibility criteria, both the first and second reviewers will conduct a comprehensive title screening. Eligible publications will be exported using Endnote management software. Articles duplication will be checked using the EndNote program.

Table (2) below presents how publications will be recorded. Full articles and abstracts of studies will be screened according to the eligible criteria. The authors will consider a third reviewer of the research in case of non-accordance between the two previous reviewers. In case of difficulty to find some articles. The authors will need the assistance of the UKZN library.

Table 2. Electronic search record

\section{Keyword search: Search engine used Number of articles or publications selected}

The mainstream healthcare system, orthodox therapy, modern therapy, western medicine Traditional herbal mixtures, herbal medicines, herbal concoctions, herbal teas, herbal formulations, traditional medicines

Microbial adaptation, antimicrobial resistance,

Mutual referral, bi-directional

Concurrent use, concomitant use, simultaneous use, use in association

Bacterial, viral, microbial, pathogen

Infectious diseases, viral infectious diseases, contagious bacterial diseases

Collaboration, cooperation, coaction

However, for authors whose publications will be cited and challenging to retrieve, they will be asked for assistance through a correspondence letter. If they do not respond to the correspondence, then their articles will be excluded.

The present protocol will use the developed guideline for reporting known as the Preferred Reporting Items for Systematic Reviews and Protocol Meta-Analyses 2015 (PRISMA-P 2015). The PRISMA-P 
consists of a checklist of 17 elements designed to promote the planning and reporting of a robust systematic review protocol[21].

\section{Charting the data}

Table (3) presents the flow or the charting of included studies. A data charting form will be conceived and piloted. Variables to have to summarize the included articles are shown in Table 3.

Table 3. Form for Data charting

\begin{tabular}{|l|}
\hline Author and date \\
\hline Article or study title \\
Journal full reference \\
Aims or main research question \\
Characteristics of participants \\
Recruitment context (e.g. where participants were recruited). \\
Sampling method \\
Study method or design \\
Theoretical background \\
Data collection (what data collection methods were used?) \\
Data analysis (how was the data analyzed?) \\
Intervention \\
Intervention outcome \\
Most relevant findings \\
Conclusions \\
Comments
\end{tabular}

\section{Collating, summarizing, and reporting the findings}

This study aims to map the evidence of collaboration THPs and BHPs in reducing antimicrobial resistance and treatment failure in bacterial and viral diseases in sub-Saharan Africa and summarizing the results as found from the included studies. Following data extraction, thematic content analysis will be carried out to code the data according to the following themes: types of interactions registered in publications; causes of treatment failure, approach system and medicinal plants used by THPs to treat 
infectious diseases; barriers and facilitators towards collaboration between THPs and BHPs in the management of infectious diseases which may lead to antimicrobial resistance and; types of infectious disease not cured by western medicine. The emerging theme will also be coded. NVIVO software version 11 will be employed to assist with the coding of the themes[22].

- Coding data from the included articles

- Categorizing the codes into major themes

- Displaying the data

- Identification of critical patterns in the data and identification of subthemes

- Summarizing

\section{Quality Appraisal}

The mixed-method appraisal tool (MMAT)-Version 2011 will evaluate the quality of the included studies [23]. This tool will assess the appropriateness of the study's aim, adequacy and methodology, study design, participant recruitment, data collection, data analysis, presentation of findings, and authors' discussions and conclusions. Selected studies will be scored based on a criterion that will use a score to describe them ( $50 \%$ and above). The strength of the body of evidence in the systematic review will be assessed using GRADE.

\section{Synthesis}

The resulting themes will be analyzed and critically examined in relationship with the research questions. Reviewers will explore the meanings of the results in reference to the aim of the research and the implications of these results for the forthcoming research, practice, and policy.

\section{Discussion}

This scoping review will be carried out as the first part of a more extensive study on the evidence of collaboration between THPs and BHPs in reducing antimicrobial resistance and treatment failure in bacterial and viral diseases in sub-Saharan Africa. This review will identify types of interactions registered in publications, causes of treatment failure, approach system, medicinal plants used by THPs to treat infectious diseases, barriers and facilitators towards collaboration between THPs and BHPs, types of infectious diseases cured by western medicine. Although there is a growing acknowledgment that healthcare systems are encouraging collaboration between THPs and BHPs [24-27], there is a lack of knowledge about the partnership between THPs and BHPs about antimicrobial resistance, treatment failure, or other interactions. Also, there are challenges related to the availability of relevant full-text articles.

Articles that report on other diseases than infectious diseases will be excluded because this study is focused on the use of prescribed antimicrobials and TM in the treatment of infectious diseases. This review excludes all tasks that do not report TM's help to manage bacterial and viral infections. All the 
reports on deaths that are not caused by infectious diseases and those that are not reporting cases of interaction either in TM alone, prescribed antimicrobials alone, or in the concurrent use of both TM and prescribed antimicrobials will be excluded.

Results from this study will be of benefit to researchers by highlighting gaps in evidence that may need further investigation. Study findings will be disseminated in peer-reviewed publications.

\section{Abbreviations}

THPs: traditional healer practitioners BHPs: biomedically healthcare professionals PICOS: population, intervention, comparison, outcomes, and study setting. MMAT: Mixed Methods Appraisal Tool, HIV/AIDS: Human Immunodeficiency Virus/ Acquis immunodeficiency deficiency Syndrome, TM: Traditional Medicine.

\section{Declarations}

Ethics approval and consent to participate

Not applicable

\section{Consent for publication}

Not applicable

\section{Availability of data and material}

All data generated or analyzed during this study will be included in the published scoping review.

\section{Competing interests}

The authors declare that they have no competing interests.

\section{Funding}

This study has received no funding.

\section{Authors' contributions}

AGAM and MGK drafted this manuscript. MN and TPM-T revised the manuscript for its intellectual content, technical compliance and accepted the final version of the manuscript for publication. All four authors agreed with the final version of the manuscript.

\section{Acknowledgements}


The acknowledgements are addressed to TAMBWE Willy MUZUMBUKULWA and SULE Saka AJIBOLA, Discipline of Pharmaceutical Sciences, University of KwaZulu-Natal, for proofreading this draft.

Authors' information

${ }^{1}$ AGANZE Gloire-Aimé MUSHEBENGE, is a PhD student in the Discipline of Pharmaceutical Sciences and DST-NRF CIKS Research Associate, School of Health Sciences, Westville Campus, University of KwaZuluNatal, P B X54001, Durban 4000, South Africa.

\section{References}

1. Organization $\mathrm{WH}$ : WHO guidelines on developing consumer information on proper use of traditional, complementary and alternative medicine. 2004.

2. Qi Z, Kelley E: The WHO traditional medicine strategy 2014-2023: a perspective. Science 2014, 346(6216):S5-S6.

3. Ledingham JG, Warrell DA: Concise Oxford textbook of medicine: Oxford university press; 2000.

4. Hunt G, Ledwaba J, Salimo A, Kalimashe M, Singh B, Puren A, Morris L: Surveillance of transmitted HIV-1 drug resistance in 5 provinces in South Africa in 2011. Communicable Diseases Surveillance Bulletin 2013, 11(4):122-124.

5. Lathers CM: Clinical pharmacology of antimicrobial use in humans and animals. The Journal of Clinical Pharmacology 2002, 42(6):587-600.

6. Richter M: Traditional healing and human rights in South Africa. In: XV International AIDS Conference, Bangkok: 2004; 2004: 11-16.

7. Van Wyk B-E, Gericke N: People's plants: A guide to useful plants of Southern Africa: Briza Publications; 2000.

8. Street R, Stirk W, Van Staden J: South African traditional medicinal plant trade-challenges in regulating quality, safety and efficacy. Journal of Ethnopharmacology 2008, 119(3):705-710.

9. Peltzer K: An investigation into the practices of traditional and faith healers in an urban setting in South Africa. Health SA Gesondheid 2001, 6(2):3-11.

10. Ballabh B, Chaurasia O, Ahmed Z, Singh SB: Traditional medicinal plants of cold desert Ladakhused against kidney and urinary disorders. Journal of ethnopharmacology 2008, 118(2):331-339.

11. de Wet H, Nkwanyana MN, van Vuuren SF: Medicinal plants used for the treatment of diarrhoea in northern Maputaland, KwaZulu-Natal Province, South Africa. Journal of ethnopharmacology 2010, 130(2):284-289.

12. York $\mathrm{T}$, De Wet $\mathrm{H}$, Van Vuuren S: Plants used for treating respiratory infections in rural Maputaland, KwaZulu-Natal, South Africa. Journal of ethnopharmacology 2011, 135(3):696-710. 
13. Sadarangani SP, Estes LL, Steckelberg JM: Non-anti-infective effects of antimicrobials and their clinical applications: a review. In: Mayo Clinic Proceedings: 2015: Elsevier; 2015: 109-127.

14. Fennell C, Lindsey K, McGaw L, Sparg S, Stafford G, Elgorashi E, Grace O, Van Staden J: Assessing African medicinal plants for efficacy and safety: pharmacological screening and toxicology. Journal of Ethnopharmacology 2004, 94(2):205-217.

15. Pretorius E: Traditional and modern medicine working in tandem. Curationis 1991, 14(4):10-13.

16. Busia K, Kasilo OM: Collaboration between traditional health practitioners and conventional health practitioners: some country experiences. Afr Health Monit 2010, 13.

17. Kamatou G, Viljoen A, Van Vuuren S, Van Zyl R: In vitro evidence of antimicrobial synergy between Salvia chamelaeagnea and Leonotis leonurus. South African Journal of Botany 2006, 72(4):634-636.

18. Nascimento GG, Locatelli J, Freitas PC, Silva GL: Antibacterial activity of plant extracts and phytochemicals on antibiotic-resistant bacteria. Brazilian journal of microbiology 2000, 31(4):247256.

19. Colquhoun HL, Levac D, O'Brien KK, Straus S, Tricco AC, Perrier L, Kastner M, Moher D: Scoping reviews: time for clarity in definition, methods, and reporting. Journal of clinical epidemiology 2014, 67(12):1291-1294.

20. Arksey H, O'Malley L: Scoping studies: towards a methodological framework. International journal of social research methodology 2005, 8(1):19-32.

21. Moher D, Shamseer L, Clarke M, Ghersi D, Liberati A, Petticrew M, Shekelle P, Stewart LAJSr: Preferred reporting items for systematic review and meta-analysis protocols (PRISMA-P) 2015 statement. 2015, 4(1):1.

22. Castleberry A: NVivo 10 [software program]. Version 10. QSR International; 2012. In.: AJPE; 2014.

23. Pluye P, Robert E, Cargo M, Bartlett G, O'cathain A, Griffiths F, Boardman F, Gagnon M-P, Rousseau M: Proposal: A mixed methods appraisal tool for systematic mixed studies reviews. Montréal: McGill University 2011:1-8.

24. Bedwell WL, Wildman JL, DiazGranados D, Salazar M, Kramer WS, Salas E: Collaboration at work: An integrative multilevel conceptualization. Human Resource Management Review 2012, 22(2):128-145.

25. Kozlowski SW, Chao GT, Grand JA, Braun MT, Kuljanin G: Advancing multilevel research design: Capturing the dynamics of emergence. Organizational Research Methods 2013, 16(4):581-615.

26. Nancarrow SA, Booth A, Ariss S, Smith T, Enderby P, Roots A: Ten principles of good interdisciplinary team work. Human resources for Health 2013, 11(1):19.

27. Walsh JA, Warren KS: Selective primary health care: an interim strategy for disease control in developing countries. Social Science \& Medicine Part C: Medical Economics 1980, 14(2):145-163.

\section{Figures}




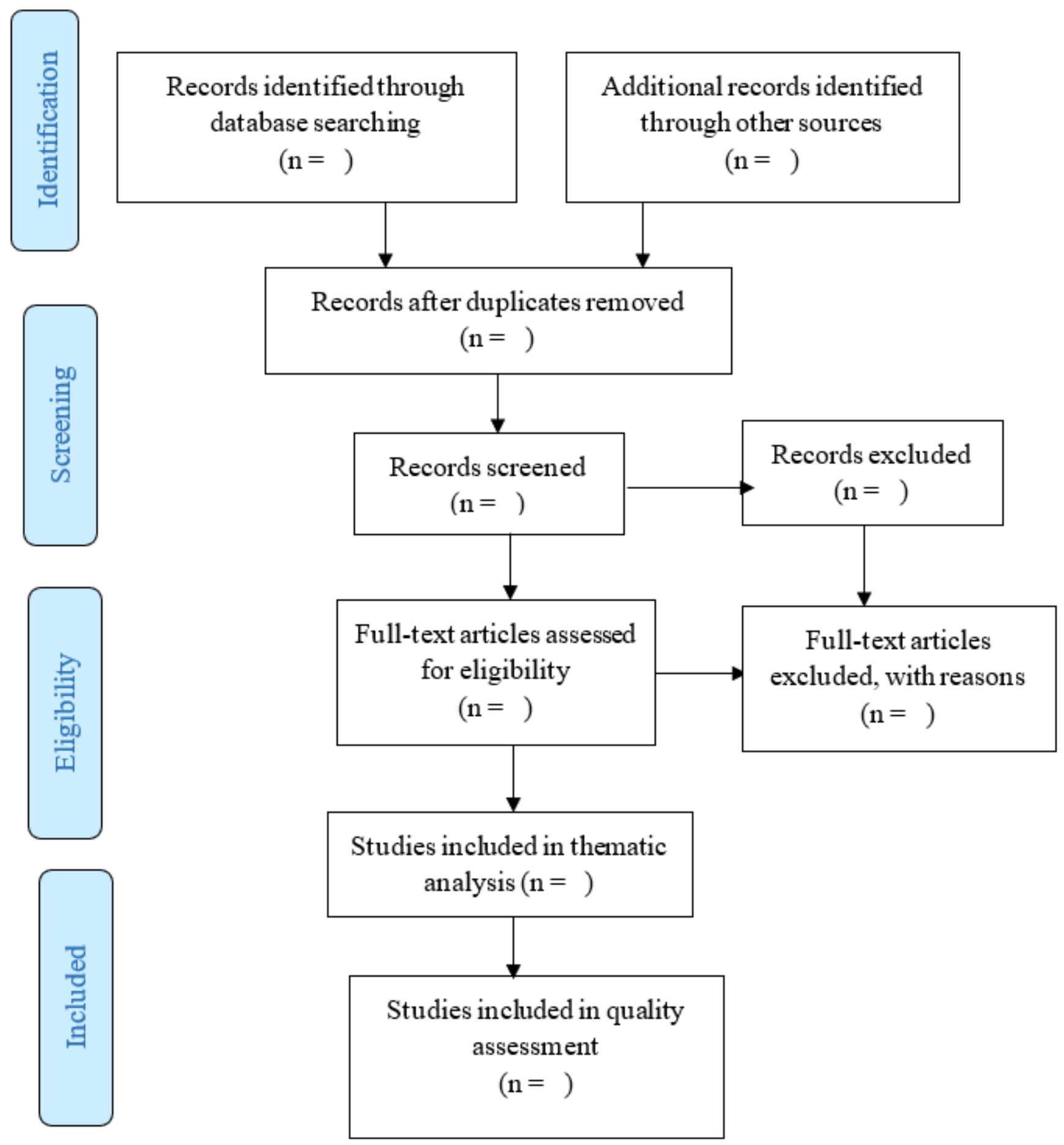

Figure 1

Illustration of the PRISMA Flow Diagram for the selection of studies.

\section{Supplementary Files}

This is a list of supplementary files associated with this preprint. Click to download. 
- Appendix1Searchengine.docx

- PRISMAPCHECKLISTPROTOCOL04012021.docx 